\title{
Electron Tomography of Quantum Dot Bioconjugates
}

\author{
A.M. Courtis, ${ }^{*}$ R. Mazitschek, ${ }^{* *}$ and D.C. Bell*** \\ * Department of Chemistry \& Chemical Biology, Harvard University, Cambridge, MA \\ ** Center for Systems Biology, Massachusetts General Hospital, Boston, MA \\ *** School of Engineering and Applied Sciences, Harvard University, Cambridge, MA
}

Quantum dots (QDs) are single nanocrystals ( 1-10nm) that are most commonly comprised of Group II-VI semiconductors. Since the 1980s there has been significant research into the unique electronic and structural attributes of QDs. Electron microscopy, in particular, has played an central role in the characterization and verification of inorganic QD synthesis [1,2]; an example of Si QDs is shown in Fig. 1. From the analysis of one of the first core-shell QD systems, $\mathrm{HgS} / \mathrm{CdS}$ [3], to more recent studies utilizing $\mathrm{Z}$-contrast $\mathrm{C}_{\mathrm{s}}$-STEM to examine epitaxial $\mathrm{ZnS}$ coating on $\mathrm{CdSe}$ quantum dots[4], EM has been invaluable in the applied and theoretical study of QD chemistry.

More recently, there has been great interest in biomedical research towards using QDs as a new class of fluorophores for both in vitro and in vivo studies of (human) physiology and disease biology. In particular, the brightness, photostability, and tunable emission profiles of QDs, which far surpass organic dyes, are attractive features. The model system for such applications would be a QD conjugated to small molecule ligands and biomacromolecules such as proteins, nuleic acids and carbohydrates (Fig 2). While such systems have been reported on in the literature [5], unambiguous characterization on the molecular level of protein association and the nature of the bioconjugate structure is still lacking. Resported models have, to this point, been based on indirect evidence. Though it is not uncommon to use colloidal gold in the EM of biological samples, either for positioning during tilt series, antigen labeling, or other applications, this knowledge has not lead to the study QD-bioconjugates as individual structures of interest. While QDs have been explored in EM vis-à-vis immunohistochemistry applications, they have not been examined as unique structures at high resolution. In fact, to our knowledge, QD-protein conjugates have yet to be studied as EM samples in their own right.

In this paper, we present the great potential electron microscopy holds for the analysis of complex, QD-based biological probes. We begin our characterization with the facile analysis of the inorganic, crystalline CdSe/ZnS QDs (Fig 3). Then, via electron tomography, we reconstruct the three-dimensional structure of $\mathrm{CdSe} / \mathrm{ZnS}$ protein bioconjugates, synthesized both via commercial precursors as well as the author's own 'bottom-up' methods. The characterization of these bioconjugates provides valuable insights towards the attachment geometries and whether structural changes are induced in proteins upon QD association. Rigorous analysis further allows for the confirmation of the average number of biological binding partners per QD. This approach yields more complete characterization of QD-bioconjugates than is presently employed in the field. It further highlights new possibilities, as well as challenges, for electron tomography in nanoscience.

\section{References}

1. A.P. Alivasatos, J. Phys. Chem. 31 (1996) 13226-13239.

2. S.J. Rosenthal et al., Surface Science Reports 62 (2007) 111-157.

3. H. Weller Advanced Materials 5 (1993) 88-95.

4. J. McBride et al., Nano Letters 6 (2006) 1496-1501.

5. H. Mattoussi et al., JACS 122 (2002) 12142-12150. 


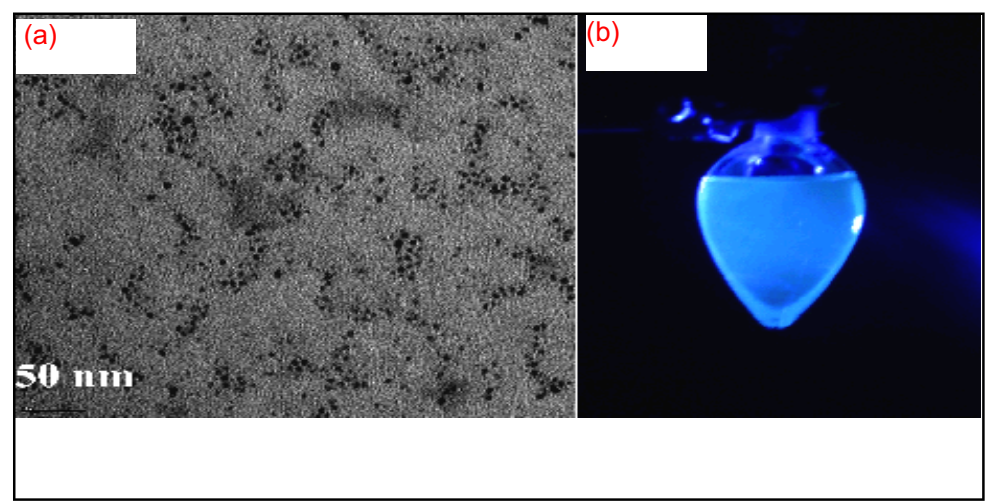

Fig. 1: (a) Si Quantum Dots

b) Representative optical properties of QDs (UV illumination)

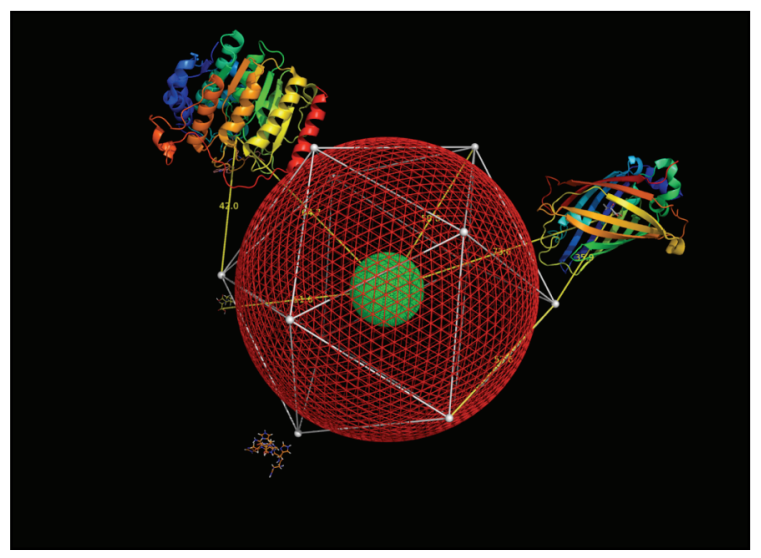

Fig. 2: Model of QD-bioconjugate (A.M. Courtis \& R. Mazitschek)

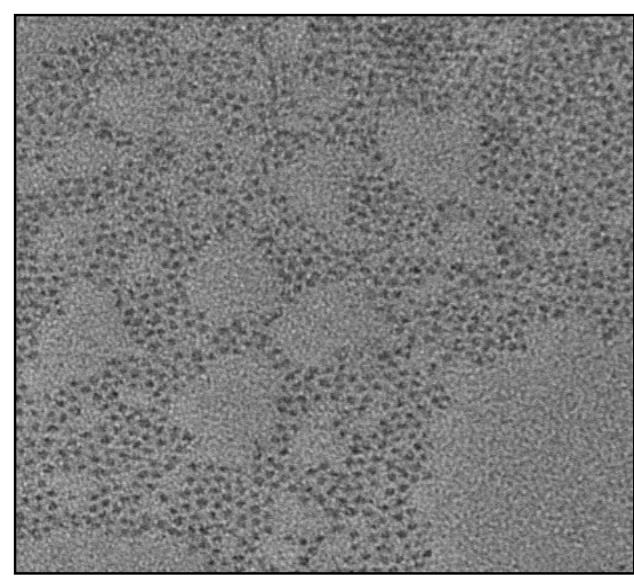

Fig. 3: CdSe/ZnS@80,000x, JEOL 2100; $200.0 \mathrm{kV}$ 